\title{
A Dual-Broadband Circularly Polarized Slot Antenna for RFID Reader Applications
}

\author{
Hao Xie, Manxi Wang \\ State Key Lab of Complex Electromagnetic Environment \\ Effects on Electronic and Information System, \\ Luoyang, 471003 China \\ E-mail: xiehao@coer-zju.org
}

\author{
Yusha Liu, Jun Hu \\ Centre for Optical and Electromagnetic Research, \\ Zhejiang University, \\ Hangzhou, 310058 China \\ E-mail: hujun@zju.edu.cn
}

\begin{abstract}
In this article, a compact RFID reader antenna operated on UHF band and WLAN with circular polarization is proposed. The antenna is comprised of an inverted L-shaped strip and an asymmetric $U$-shaped strip. The antenna is fed by a coplanar waveguide (CPW) line. The simulated result for -10 -dB impedance bandwidth is about $550 \mathrm{MHz}(875-1425 \mathrm{MHz})$ and $480 \mathrm{MHz}(2.16-2.64 \mathrm{GHz})$. Its corresponding simulated 3-dB axial-ratio (AR) bandwidth is about $398 \mathrm{MHz}(772-1170 \mathrm{MHz})$ and $55 \mathrm{MHz}(2.32-2.375 \mathrm{GHz})$. The overall antenna size is $120 \mathrm{~mm}$ $\times 120 \mathrm{~mm} \times 1.6 \mathrm{~mm}$. The results show a perfect circularly polarized radiation in both the lower and upper bands. In comparison to the recent works, the proposed antenna has wider impedance bandwidths, and better circularly polarized performance.
\end{abstract}

Keywords—circular polarization;slot antenna;dual-band; RFID reader.

\section{INTRODUCTION}

In recent years, applications for radio frequency identification (RFID) systems has been received much interest in several service industries, such as, purchasing, distribution logistics, and animal tracking applications [1]. And dual frequency bands at $915 \mathrm{MHz}$ and $2.45 \mathrm{GHz}$ are popularly used in RFID UHF band and WLAN. For a commercial RFID system, it usually consists of a reader and many tags. In this article, the RFID reader antenna is designed as circular polarization radiation pattern in order to increase the orientation diversity. Thus, it is able to receive any linearly polarized (LP) RFID tag energy which is popularly employed in RFID system.

Currently, the antennas for the RFID reader should be characterized by high-quality circular polarization characteristic, wide impedance bandwidth and high gain [2]. In order to achieve these goals, [3]-[5] have presented multilayer or multi-feed configurations. In [3], a dual-band circularly polarized (CP) microstrip antenna for satellite applications is proposed. For this antenna, the measured bandwidths for a VSWR less than 2.0 are about $1.5 \%$ (30 $\mathrm{MHz})$ at $1.7 \mathrm{GHz}$ and $8.64 \%(700 \mathrm{MHz})$ at $8.1 \mathrm{GHz}$. This antenna can satisfy two Meteosat bandwidth goals, but it was realized with a 1.5-mm-thick upper layer substrate and a 0.758-mm-thick feed layer substrate. A dual-band circularly polarized antenna using stacked patches with asymmetric U- slots is presented in [4]. The circular polarization radiation pattern is achieved by designing asymmetrical U-slots on the patches and the antenna has three layers. The circularly polarized bandwidth of the antenna is $1.0 \%$ at $3.5 \mathrm{GHz}$ (WiMax) and $3.1 \%$ at $5.8 \mathrm{GHz}$ (HiperLAN). The measured $\left|S_{11}\right|$ bandwidths of the antenna are about $3.0 \%$ at the lower band and $14.9 \%$ at the upper band. In [5], a dual-band circularly polarized S-shaped slotted patch antenna is proposed. For this antenna, the measured $10-\mathrm{dB}$ return loss bandwidths for the lower and upper-bands are 16\% (1.103$1.297 \mathrm{GHz})$ and $12.5 \%(1.444-1.636 \mathrm{GHz})$, respectively. The measured 3-dB axial-ratio (AR) bandwidth is 6.9\% (1.195$1.128 \mathrm{GHz}$ ) for the lower-band and $0.6 \%(1.568-1.577 \mathrm{GHz})$ for the upper-band. And the antenna has a thickness of $23 \mathrm{~mm}$. It is difficult for slot CP antennas to achieve broad bandwidths in both impedance and AR at two operating bands using a single-layer and single-feed configuration. In this paper, a single-layer and single-feed RFID reader antenna with circularly polarized radiating pattern is proposed. The wide axial-ratio bandwidth can obtain by tuning the inverted Lshaped strip and the asymmetric U-shaped strip at two frequency bands. Specifically, the reader antenna is compact with a relatively small size of $120 \mathrm{~mm} \times 120 \mathrm{~mm} \times 1.6 \mathrm{~mm}$.

\section{Antenna Design PROCESS}

The final structure and dimensions of the proposed compact circularly polarized reader antenna is shown in Fig. 1. The antenna is fabricated on a FR4 substrate $\left(\varepsilon_{\mathbf{r}}=4.4\right.$, $\tan \delta=0.02)$ and has a thickness of $H(H=1.6 \mathrm{~mm})$. The antenna radiating patch has a length of $G(G=120 \mathrm{~mm})$, and the square slot has length of $L(L=89 \mathrm{~mm})$. The antenna is fed by a coplanar waveguide (CPW) line located at the lower left corner of the structure. The CPW line has a $50 \Omega$ characteristic impedance with a length of $L_{1}$ and a width of $W_{1}$. The CPW line has a gap of $g$ with the radiating patch. An inverted L-shaped strip used for impedance matching and circularly polarized at lower band is connected to the end of the CPW line. The inverted L-shaped strip has a width of $W_{2}$. An asymmetric U-shaped strip is embedded at the lower of the square slot. The $G$ and $L$ are fixed with a $G / L$ ratio of 1.35 in this article. The dimensions of antenna are listed in Table I. All metal elements are printed on the top of the substrate. 


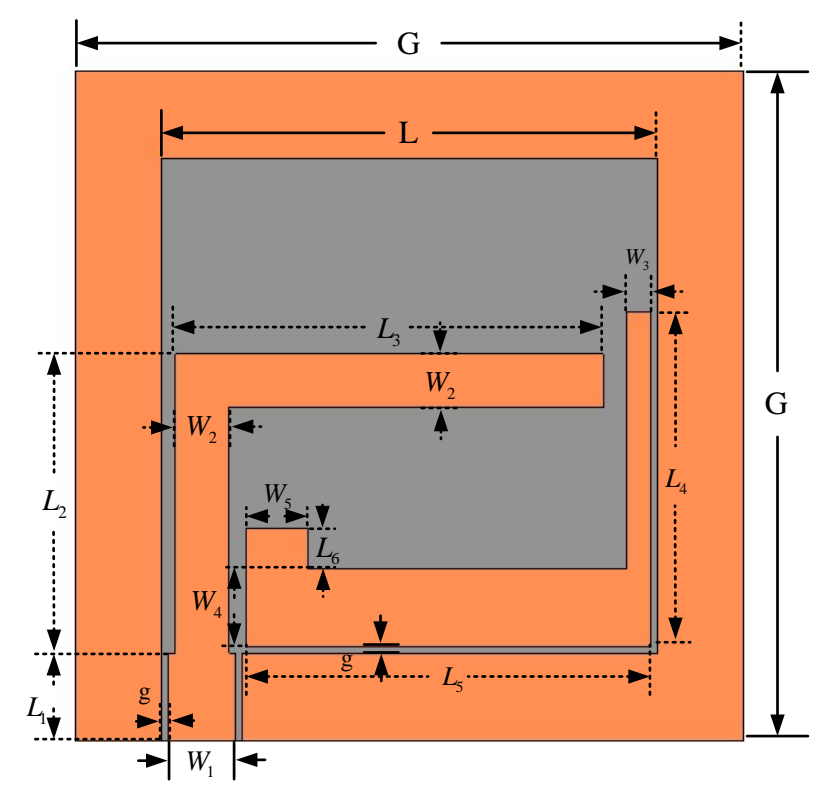

Fig. 1. Geometry of the circularly polarized antenna

TABle I. The Parameters For The Proposed ANTENNA

\begin{tabular}{|c|c|c|c|c|c|}
\hline Para. & Value & Para. & Value & Para. & Value \\
\hline$G$ & $120 \mathrm{~mm}$ & $L_{2}$ & $53.8 \mathrm{~mm}$ & $W_{3}$ & $4.3 \mathrm{~mm}$ \\
\hline$L$ & $88.8 \mathrm{~mm}$ & $L_{3}$ & $76.8 \mathrm{~mm}$ & $L_{5}$ & $73.5 \mathrm{~mm}$ \\
\hline$L_{1}$ & $15.6 \mathrm{~mm}$ & $W_{2}$ & $9.6 \mathrm{~mm}$ & $W_{4}$ & $16.1 \mathrm{~mm}$ \\
\hline$W_{1}$ & $12 \mathrm{~mm}$ & $L_{4}$ & $46.1 \mathrm{~mm}$ & $L_{6}$ & $7.2 \mathrm{~mm}$ \\
\hline$W_{5}$ & $11 \mathrm{~mm}$ & $G$ & $1.2 \mathrm{~mm}$ & $/$ & $/$ \\
\hline
\end{tabular}

In the proposed antenna, the inverted L-shaped strip and the asymmetric U-shaped strip are key components to excite the required 90 degree phase difference for a $\mathrm{CP}$ radiation. In order to design the required $\mathrm{CP}$ antenna, firstly, the antenna size $G$ is designed about half wave-length at the lower band. Then, the impedance matching and $\mathrm{CP}$ radiating pattern at lower band is achieved by adjusting the inverted L-shaped strip dimensions $L_{2}, L_{3}$ and $W_{2}$. And the impedance matching and $C P$ radiation at upper band is achieved by adjusting the $\mathrm{U}$ shaped strip dimensions $L_{4}, L_{5}, L_{6}, W_{3}, W_{4}$ and $W_{5}$. Finally, the dimensions of the proposed antenna are slightly modified to optimize the circular polarization. The simulated impedance bandwidth which is determined by the $-10 \mathrm{~dB}$ reflection coefficient and the $\mathrm{CP}$ bandwidth which is determined by $3 \mathrm{~dB}$ AR are presented in Fig. 2 and Fig. 3. The simulated results are presented by tuning the length $L_{5}$ from $71 \mathrm{~mm}$ to $73 \mathrm{~mm}$, with $1 \mathrm{~mm}$ step increment. As shown in Fig. 2 and Fig. 3, the impedance bandwidth can be slightly increased by increasing parameter $L_{5}$ and a small value of $L_{5}$ will improve $\mathrm{CP}$ bandwidths at both lower and upper band. In Fig. 2, the bandwidth in reflection coefficient and AR for the lower band is wider because it will produce two adjacent modes.

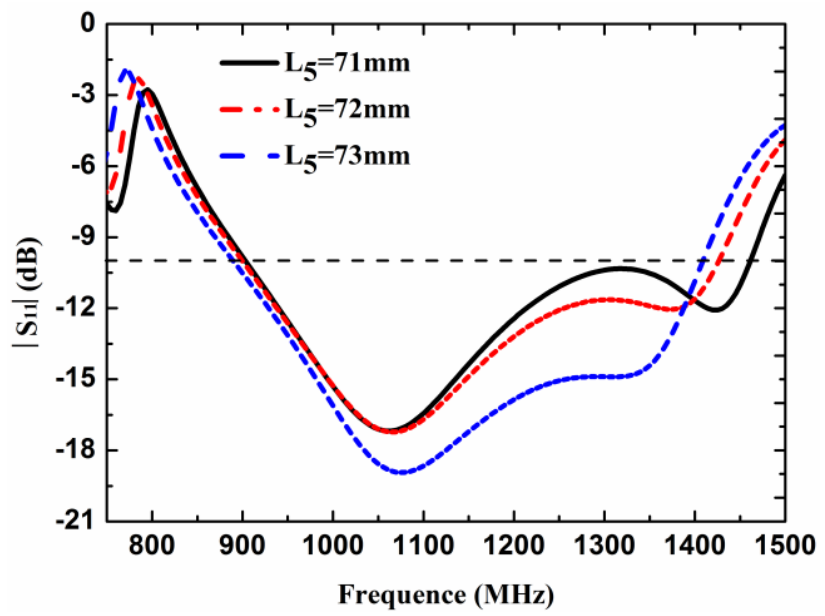

(a)

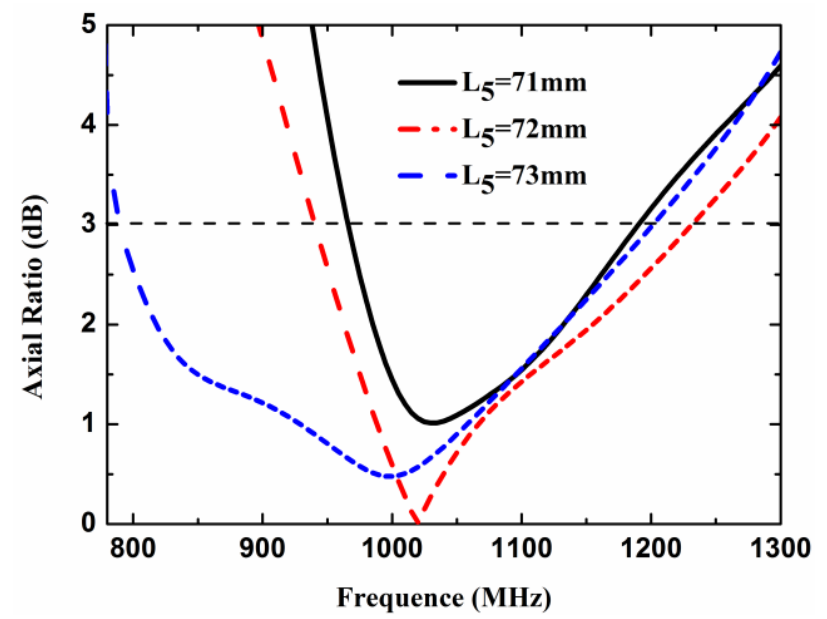

(b)

Fig. 2. (a) Reflection coefficient and (b) axial ratio of the proposed CP antenna at lower band with varying of $L_{5}$, the location of the horizontal arm of the U-shaped strip

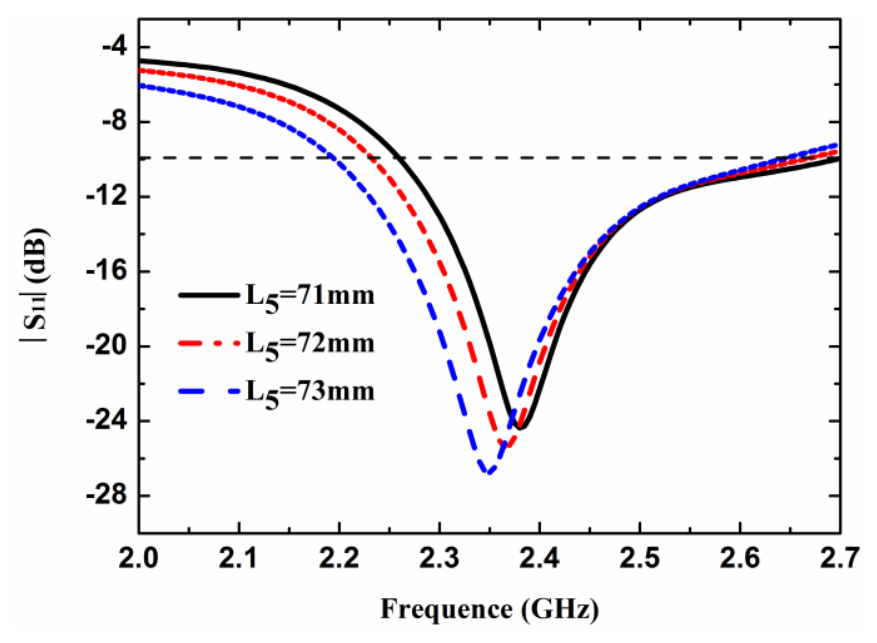

(a) 


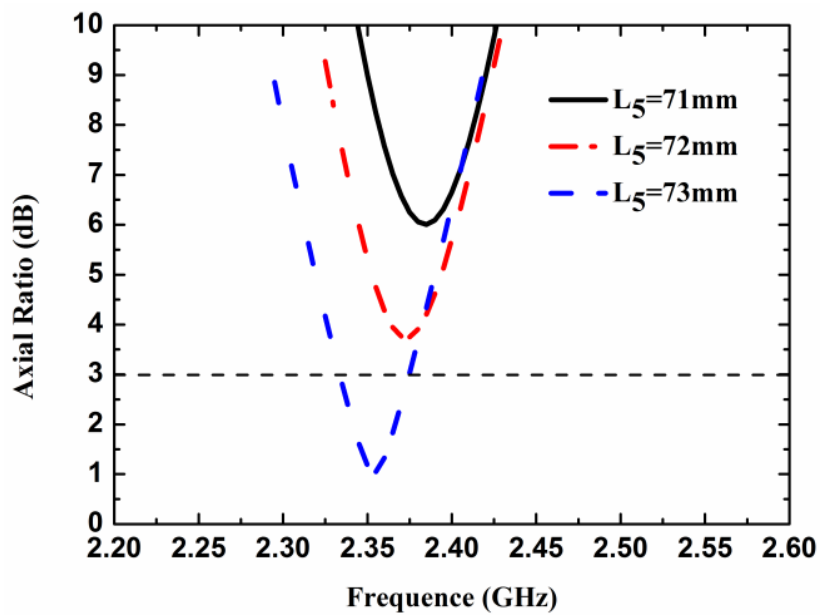

(b)

Fig. 3. (a) Reflection coefficient and (b) axial ratio of the proposed CP antenna at upper band with varying of $L_{5}$, the location of the horizontal arm of the U-shaped strip

\section{RESULTS AND DISCUSSIONS}

Note that the commercially available simulation software, Ansoft High Frequency Simulator Structure (HFSS) 13 is used for the simulated results. The lower band simulated results when $L_{5}=73.5 \mathrm{~mm}$ are presented in Fig. 4, and the upper band simulated results are presented in Fig. 5. The simulated impedance bandwidths $(-10 \mathrm{~dB}$ reflection coefficient) at the lower and upper bands are 60\% (875-1425 MHz) and 20\% (2.16-2.64 GHz), respectively. As shown in Fig. 4 and Fig. 5, the simulated impedance bandwidth at the lower is better than the upper. It almost covers the whole UHF RFID band which ranges from 860 to $960 \mathrm{MHz}$. The antenna can be considered as a broadband antenna since its impedance bandwidths at two operating bands are larger than $20 \%$. With the parameters as shown in Table I, the simulated circularly polarized bandwidths determined by the $3-\mathrm{dB}$ axial ratio, are $398 \mathrm{MHz}$ $(772-1170 \mathrm{MHz})$ at lower band and $55 \mathrm{MHz}(2.32-2.375 \mathrm{GHz})$ at the upper band, as shown in Fig. 4 and Fig. 5 . At the lower band, the AR is best at $1 \mathrm{GHz}$ with $A R=0.47 \mathrm{~dB}$. And at the upper band, the AR is best at $2.345 \mathrm{GHz}$ with $A R=0.23 \mathrm{~dB}$. That illustrated the proposed tag antenna has an excellent $C P$ radiation. The maximum simulated gains for the proposed antenna are about $2.5 \mathrm{~dB}$ at $900 \mathrm{MHz}$ and $2.8 \mathrm{~dB}$ at $2.3 \mathrm{GHz}$, and the simulated radiation efficiency are about $98.8 \%$ at 900 $\mathrm{MHz}$ and $98.8 \%$ at $2.3 \mathrm{GHz}$. The antenna radiates electromagnetic fields with a left-hand circular polarization (LHCP) in the direction $Z>0$ and a right-hand circular polarization (RHCP) in the direction $Z<0$ at both the lower band and upper band. With further adjustment for the inverted L-shaped strip and the asymmetric U-shaped strip dimensions, such as, $L_{2}, L_{3}, W_{2} L_{4}, L_{5}, L_{6}, W_{3}, W_{4}$ and $W_{5}$, the circularly polarized bandwidth at the upper band can be improved and the antenna performance will be better.

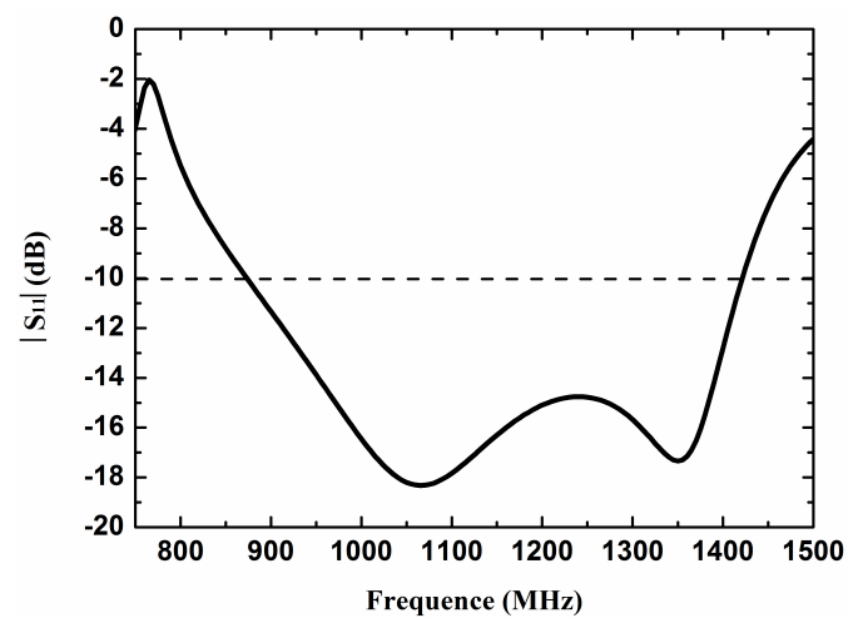

(a)

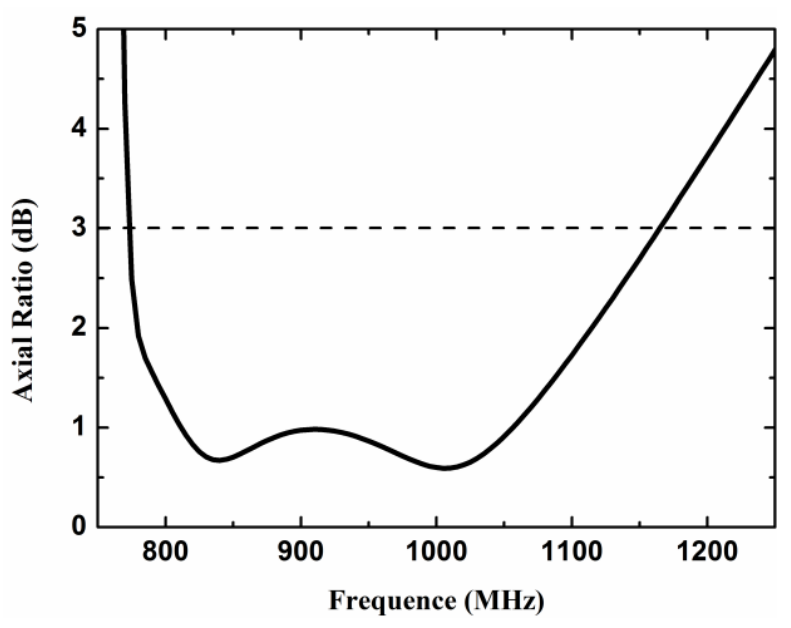

(b)

Fig. 4. (a) Reflection coefficient and (b) axial ratio of the proposed CP antenna at lower band

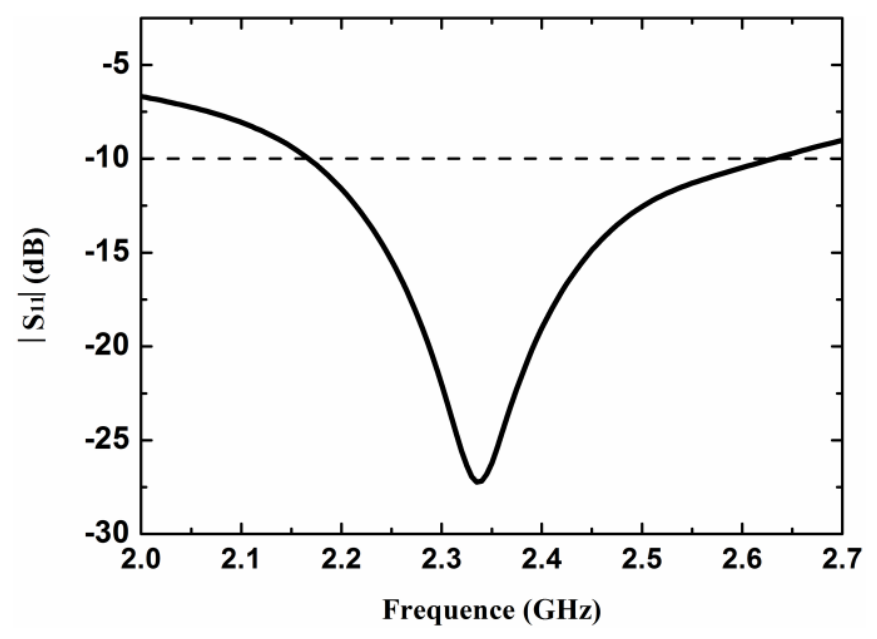

(a) 


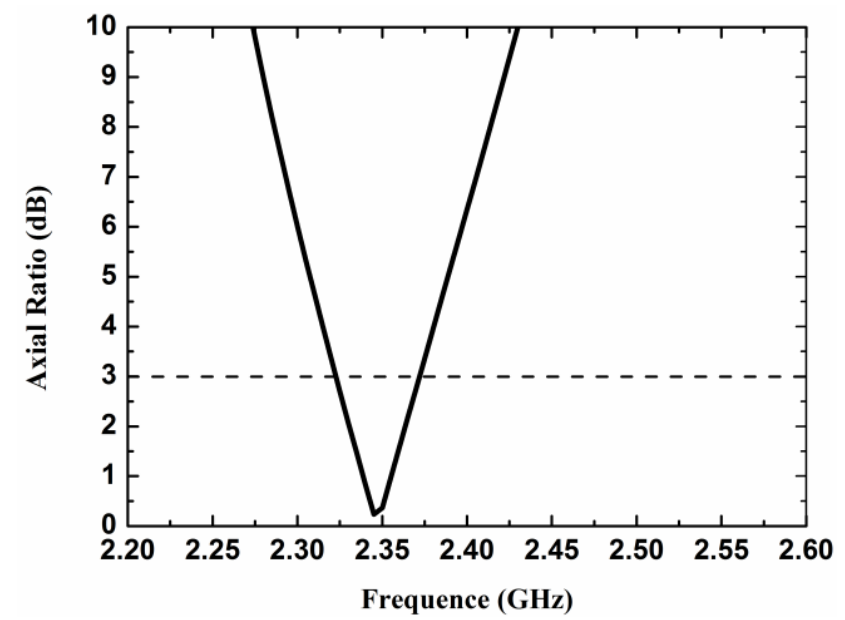

(b)

Fig. 5. (a) Reflection coefficient and (b) axial ratio of the proposed CP antenna at upper band

\section{CONCLUSION}

A compact dual-band RFID reader antenna with excellent circular polarization and impedance bandwidth has been designed. The circularly polarized radiation pattern and perfect impedance matching obtained in this paper are determined by the inverted L-shaped strip and the asymmetric
U-shaped strip. The simulated results have been shown that the antenna has a wider impedance bandwidth and circular polarization bandwidth. The simulated results verify a good $\mathrm{CP}$ radiation at two frequency band. With low profile, lower weight, and lower cost, the proposed reader antenna is a good candidate for RFID UHF band and WLAN.

\section{Acknowledgment}

The work was supported by the State Key Lab of Complex Electromagnetic Environment Effects on Electronic and Information System.

\section{References}

[1] K. Finkenzeller, RFID Handbook, 2nd ed. New York: Wiley \& Sins, 2004.

[2] W. Son, H. Lee,M. Lee, S.Min, and J. Yu, "Compact square quadrifilar spiral antenna with circular polarization for UHFmobile RFID reader," in Proc. Asia-Pacific Microw. Conf., 2010, pp. 2271-2274.

[3] F. Ferrero, C. Luxey, G. Jacquemod, and R. Staraj, "Dual-band circularlypolarized microstrip antenna for satellite applications," IEEE Antennas Wireless Propag. Lett, vol. 4, pp. 13-15, 2005.

[4] P. Nayeri, K. F. Lee, A. Z. Elsherbeni, and F. Yang, "Dualbandcircularly polarized antennas using stacked patches with asymmetric U-slots," IEEE Antennas Wireless Propag. Lett, vol. 10, pp. 492-495, 2011.

[5] Nasimuddin, Z. N. Chen, and X. M. Qing, "Dual-band circularly polarized S-shaped slotted patch antenna with a small frequency-ratio," IEEE Trans. Antennas Propag, vol. 58, no. 6, pp. 2112-2115, Jun. 2010. 